\title{
Neuroprotection against neurodegenerative disorders by histone acetyltransferase inhibitors: An overview
}

\author{
Walter Milano', Mario F Tecce ${ }^{2}$ and Anna Capasso ${ }^{2}$ \\ ${ }^{1}$ Mental Health Operational Unit District 24 ASL Naples 1 Center, Italy \\ ${ }^{2}$ Department of Pharmacy, University of Salerno, Fisciano 84084, Italy
}

\begin{abstract}
Histone acetyltransferase inhibitors are able to induce neuroprotection because of their antioxidant properties, so, their therapeutic use in limiting neurological damage is strongly supported. Histone acetyltransferase inhibitors treatments should not only aim to alleviate specific symptoms but also attempt to delay/arrest disease progression and to repair the damaged structures. The acetylation and deacetylation of histones are involved in the regulation of gene expression and the regular function of histone acetyltransferases (HATs) and deacetylases (HDACs) provide regulatory steps for gene expression and cell cycle. Functional defects of these enzymes may lead to several diseases, including neurodegenerative disorders. The studies reported in the present review support the view that the histone acetyltransferase inhibitors may be a key modulatory element in the control of neurodegenerative disorders. This idea is supported by different anatomical, electrophysiological, pharmacological and biochemical data. Furthermore, these studies support the idea of developing novel pharmacotherapies with histone acetyltransferase inhibitors that selectively target specific area of CNS.
\end{abstract}

\section{Introduction}

Although several genetic and molecular mechanisms have been proposed for neurodegenerative disorders, the mechanisms underlying such diseases remains still unclear.

Epigenetic modifications in the etiology of neurodegenerative diseases have also been considered thus indicating an interesting approach for therapeutic interventions [1-6]. The epigenetic changes do not modify nucleotide sequences in the DNA but influence its functioning by controlling its expression by gene reprogramming [2]. Epigenomic variation leads to phenotypic diversity as well as susceptibility of individuals to disease. DNA methylation and histone acetylation have been recognized as epigenetic processes which regulate the functioning of gene. Histone acetylation controls the heterochromatic and euchromatic state of DNA wrapped around histones, and remaining in dormant state. Histone acetylation unwinds the DNA from histone and renders it available for transcription. Along with histone acetylation, DNA methylation plays an important role in regulating accessibility of DNA for transcription. Histone acetylase transferases (HAT) and Histone deacetylase (HDAC) controls histone modification in cell [3]. [4-6]:

Histone modifications regulate gene expression in different ways

1) by regulating chromatin structure

2) by integrating responses to multiple biochemical signaling cascades

3) by mediating epigenetic changes in gene expression

Histone acetyltransferases, are a family of enzymes that acetylate the histone tails of the nucleosome. This, and other modifications, is expressed based on the varying states of the cellular environment [4-7]. Many proteins with acetylating abilities have been documented and, after a time, were categorized based on sequence similarities between them. These similarities are high among members of a family, but members from different families show very little resemblance [4-7].

Also, there are a total of four classes that categorize Histone Deacetylases (HDACs). Class I includes HDACs 1, 2, 3, and 8. Class II is divided into two subgroups, Class IIA and Class IIB. Class IIA includes HDACs 4, 5, 7, and 9 while Class IIB includes HDACs 6 and 10. Class III contains the Sirtuins and Class IV contains only HDAC11[8,9]. Classes of HDAC proteins are divided and grouped together based on the comparison to the sequence homologies of Rpd3, Hos1 and Hos2 for Class I HDACs, HDA1 and Hos3 for the Class II HDACs and the sirtuins for Class III HDACs [8,9].

The balance between HAT and HDAC levels is a key modulatory element for establishing appropriate histone modification patterns that serve to regulate both stable and rapidly changing gene expression profiles critical for both neuronal homeostasis, and neurophysiological response outputs [10]. Therefore, these studies support the maintenance of optimal HAT/HDAC balance for neuronal survival as they play a dynamic role in switching a gene ON and OFF $[11,12]$. Dysfunction and deregulation of these enzymes leads to aberrant expression of genes thereby affecting the cellular phenotype, resulting in diseases like neurodegenerative disorders [11-13]. Recently, it has been shown that inhibition of histone deacetylase enzymes (HDACs) induces neuroprotective effects in neurodegenerative disorders $[14,15]$. The protective effects of HDAC inhibitors seems to be related to a restabilization of gene transcription, owing to a shift of histone acetylation

Correspondence to: Anna Capasso, Department of Pharmacy, University of Salerno, Fisciano 84084, Italy annacap@unisa.it

Key words: histone acetyltransferase inhibitors, neurodegenerative disorders

Received: October 21, 2017; Accepted: November 13, 2017; Published: November 16, 2017 
equilibrium towards increased acetylation of histones, relaxation of DNA-chromatin complexes and subsequent increase of gene transcription. This observation support the idea that neurodegenerative disorders may be related to dysfunctions of methylation and histone modifications [14-17] thus indicating that epigenetic modifications may play a significant role in the etiology of neurodegenerative diseases and pathogenetic mechanisms involved in their propagation and may also present a great opportunity for therapeutic interventions [14-17].

Given the above evidences, the studies reported in the present review support the view that the histone acetyltransferase inhibitors may be a key modulatory element in the control of neurodegenerative disorders.

\section{HDAC inhibitors}

Recent years have witnessed an explosion in the development of new HDAC inhibitors, a class of compounds that intervene with the function of histone deacetylase by displacing the $\mathrm{Zn}^{++}$. Without $\mathrm{Zn}^{++}$ HDAC cannot recognize the acetyle group and unable to remove it from histone leading to euchromatin formation. First HDAC inhibitors discovered were naturally occurring microbial metabolites such as TSA (trichostatin A, isolated from Streptomyces hygroscopicus) and the depsipeptide FK228 (isolated from Chromobacterium violaceum). While TSA is a hydroxamic acid-based pan-HDAC inhibitor, FK228 is a natural cyclic peptide which selectively inhibits HDACs 1 and 2 . Hydroxamic acid is known as one of the most potent zinc-chelating group due to its capability to reproduce the natural planar square structure of the zinc ion in the catalytic pocket of HDAC enzymes.

Other naturally occurring HDACi have been isolated from fungi (e.g., depudecin and trapoxin A and B) and marine life (e.g., largazole and azumamides) [18]. Given their rich source of biologically active compounds, extensive efforts have also been undertaken to identify novel plant-derived HDACi. Allyl derivatives from garlic were among the first compounds described to impact histone acetylation status [18]. Other well-characterized plant HDACi include sulforaphane (SFN) isolated from cruciferous vegetables, and quercetin, found in a variety of fruit. Given the promise of HDACi to treat a variety of diseases, particularly cancer, there is growing interest in the potential of dietary compounds that possess HDAC inhibition activity. Some other natural compounds including ancardic acid, curcumin and garcinol, described as inhibitor of HAT activity of p300/CBP, an enzyme that plays a central role in different cellular processes. Recently, we reported that a series of oxidized and polyisoprenylated benzophenone derivatives isolated from plants of the family Clusiaceae, in particular those belonging to the genera Clusia, and Garcinia, are potent modulator of histone acetyltransferases. In particular clusianone, guttiferone A and guttiferone $\mathrm{E}$ inhibit the p300 HAT activity, whereas nemorosone showed a great ability to activate the enzyme due to its high affinity for the p300/CBP enzyme [19]. From a biogenetic point of view, polyisoprenylated benzophenone derivatives are thought to be of mixed shikimate and acetate biosynthetic origin in which the acetate derived benzene ring is modified by intervention of prenyl groups. Structural modifications, oxidation and alkylation of this ring results in the formation of a complex bridged bicyclic or tricyclic system as bicyclo-[3.3.1]-nonane derivatives (Figure 1).

Many of the new synthetically-derived HDAC inhibitors have been designed based on structural information obtained from naturally occurring compounds. Currently, the most advanced HDAC inhibitor is a compound called SAHA (suberoylanilide hydroxamic acid; also known as vorinostat (Zolinza ${ }^{\oplus}$; Merck, Darmstadt, Germany) which was discovered through extensive surveys of small polar molecules capable of inhibiting HDAC enzymes [17].

Finally, HDAC inhibitors can be classified into four main chemical families, the short-chain fatty acids (e.g., sodium butyrate, phenylbutyrate and valproic acid), the hydroxamic acids (e.g., trichostatin A and suberoylanilide hydroxamic acid (SAHA), the epoxyketones (e.g., trapoxin) and the benzamides. Of these, the most widely studied are sodium butyrate, phenylbutyrate, trichostatin A and SAHA. The butyrates are known to cross the blood-brain barrier. The initial interest in these inhibitors came from studies linking HDACs to a wide variety of human cancers. HDAC inhibitors arrest growth, induce differentiation and, in some cases, apoptosis and have potent anit-cancer activities, with remarkable tumor specificity. For this reason, inhibitors of class 1 and 2 HDACs are in phase I/II clinical trials for cancer therapy and potentially cancer prevention. In the nervous system, the anticonvulsant and mood-stabilizing drug valproic acid was identified as an inhibitor of HDAC1, thereby linking its anti-epileptic effects to changes in histone acetylation. As described below, more recent work has revealed that inhibitors of class 1 and 2 HDACs (sirtuins) represent novel therapeutic approaches to treat neurodegenerative disorders, depression and anxiety and the cognitive deficits that accompany many neurodevelopmental disorders.

\section{Protective effects of HDAC inhibitors on neurodegener- ative disorders}

Increasing evidence suggests that epigenetic mechanisms such as DNA methylation and histone tail modifications are dynamically regulated in neurons and play a fundamental role in learning and memory processes. In addition, both global and gene-specific epigenetic changes and deregulated expression of the writer and eraser proteins of epigenetic marks are believed to contribute to the onset and progression of neurodegeneration. Studies in animal models of neurodegenerative diseases have highlighted the potential role of epigenetic drugs, including inhibitors of histone deacetylases and methyl donor compounds, in ameliorating the cognitive symptoms and preventing or delaying the motor symptoms of the disease, thereby opening the way for a potential application in human pathologydeficits [20].

\section{Alzheimer's disease}

$\mathrm{AD}$ is the most common neurodegenerative disease in Western Europe, and an important public health problem as the number of cases is increasing with aging of the population. It manifests with progressive decline in memory and intellectual abilities, impoverishment of language, disorientation and behavioral skills. The characteristic neuropathological aspects of AD are senile plaques (SP), neurofibrillary tangles (NFT), and amyloid angiopathy. Brain lesions associated with $\mathrm{AD}$ such as NFT and SP, are characterized by the presence of a broad spectrum of inflammatory mediators produced by cells residing in the brain, including neurons. Although of secondary importance compared to the fundamental cause that determines the presence of tangles and plaques, there is strong evidence that inflammation exacerbates the neuronal cell loss. Consequently, AD risk is substantially influenced by several polymorphisms in the promoter region of genes and other non-coding regions for inflammatory mediators. Alleles that support the increased expression of inflammatory mediators or alleles that favour the reduced expression of anti-inflammatory mediators are more frequent in patients with $\mathrm{AD}$ compared to controls. The polymorphisms are fairly common in the general population, so there is a strong probability that everyone will inherit one or more 
high risk alleles. $\mathrm{AD}$ also is characterized by enhanced beta-amyloid peptide (beta A) deposition along with glial activation in senile plaques, selective neuronal loss, and cognitive deficits [20,21].

Epigenetic mechanisms are also believed to play a role in $\mathrm{AD}[20,22]$ because changes in histone tail modifications have been observed in post-mortem $\mathrm{AD}$ brains $[20,23]$. In this respect, while the levels of $\mathrm{H} 3$ acetylation (leading to increased tau phosphorylation) were reduced in the temporal lobe, the levels of the histone deacetylases HDAC6 and HDAC2 (repressing genes required for learning and memory) were increased [20-25]. Several studied have confirmed the involvement of histone modifications in AD because the HDAC inhibitors (HDACi) in $\mathrm{AD}$ animal models often resulted in both prevention of cognitive deficits and memory recovery [20-28]. HDCA inhibitors as valproic acid, trichostatin A, sodium phenylbutyrate, and vorinostat that interact with zinc-dependent HDAC proteins (class I, class II, and class IV), nicotinamide that inhibits class III HDACs, and more recent compounds that selectively inhibit certain HDACs are able to improve cognition and reduce AD-like features in AD model [20]. The hypothesis that epigenetic mechanisms are involved in the altered synaptic function and memory associated with $\mathrm{AD}$ was confirmed by using trangenic mouse model of AD.

$\mathrm{AD}$ transgenic mice, treated for 4 weeks with sodium butyrate, showed an improvement in learning and memory features [29]. Another study with sodium butyrate in AD transgenic mouse confirmed the above improvement because of a link between tau phosphorylation reduction and restoration of dendritic spine density in hippocampal neurons [30]. Furthermore, in a transgenic mouse model for amyloid deposition (APP/PS1 mice), sodium butyrate improved associative memory by increasing both hippocampal histone acetylation and the expression of genes implicated in associative learning [31].

Another study performed with APP/PS1 mouse model of AD, showed that the acute treatment with a histone deacetylase inhibitor, trichostatin A (TSA), prior to training rescued both acetylated H4 levels and contextual freezing performance to wild-type values. Moreover, TSA rescued CA3-CA1 LTP in slices from APP/PS1 mice suggesting that histone deacetylase inhibitors TSA effectively counteract disease progression [32].

Several similar examples are available in the literature concerning HDACi and memory function in AD animal models, for example 2-3 weeks treatment with either sodium valproate, sodium butyrate, or vorinostat reversed contextual memory deficits in APP/PS1 mice [20,33] a 10 days treatment with entinostat, a selective inhibitor of HDAC1, reduced neuroinflammation and amyloid plaque deposition and improved behavioral impairment in APPPS1-21 mice [20,34] and a 4 week treatment with a class II inhibitor in transgenic AD mice overexpressing mutant APP, presenilin 1 and tau proteins $(3 \times \mathrm{AD}$ mice), improved memory functions and decreased $A \beta$ and phosphorylated tau levels $[20,35]$. Taken overall, those studies suggest that targeting histone modifications with HDACi can improve cognition and reduce $\mathrm{AD}$-like features in $\mathrm{AD}$ models.

\section{Parkinson disease}

While $\mathrm{AD}$ is the most prevalent neurological disorder in the aged population, $\mathrm{PD}$ is the second most common neurodegenerative disease. Several studies indicate the presence of inflammatory mediators (including TNF- $\alpha$, IL- $1 \beta$, IL-6, and interferon- $\gamma(\mathrm{IFN} \gamma)$ ) in the cerebrospinal fluid (CSF) of patients with PD as well as in the postmortem substantia nigra pars compacta in PD patient brains $[20,21,36]$.
There is an increase in the levels of proinflammatory cytokines in the CSF and nigrostriatal regions of PD brains. Furthermore, large numbers of reactive microglia are found in the substantia nigra of PD patients. These may chronically produce ROS, resulting in depletion of antioxidant stores that may jeopardize mitochondrial activity. Since aerobic respiration in mitochondria is responsible for most of the ROS produced in cells, abnormalities in these organelles may exacerbate oxidative stress $[20,21,36]$.

Recent evidence has highlighted a pathological imbalance in PD between the acetylation and deacetylation of the histone proteins around which deoxyribonucleic acid (DNA) is coiled, in favour of excessive histone deacetylation. This mechanism of adding/removing acetyl groups to histone lysine residues is one of many epigenetic regulatory processes which control the expression of genes, many of which will be essential for neuronal survival. Hence, such epigenetic modifications may have a pathogenic role in PD. It has therefore been hypothesised that if this pathological imbalance can be corrected with the use of histone deacetylase inhibiting agents then neurodegeneration observed in PD can be ameliorated [37,38].

Therefore, the epigenetics role in $\mathrm{PD}$ pathogenesis was demonstrated by inducing the histone modifications in cell cultures and animal models with mitochondrial toxins 1-methyl-4-phenylpyridinium (MPP+), paraquat, rotenone, or $\alpha$-synuclein [37]. $\alpha$-synuclein mediates neurotoxicity in the nucleus by binding directly to histone $\mathrm{H} 3$ and inhibiting histone acetylation whereas hyperacetylation by neurotoxic pesticides and paraquat leads to a dopaminergic neuronal degeneration [37].

HDACi neuroprotection against $\alpha$-synuclein-mediated toxicity has been demostrated by several studies [38-41]. In several model of PD, the toxicity of $\alpha$-synuclein was antagonized by the administration of sodium butyrate or vorinostat or sirtuin $2[39,40]$. In addition, valproic acid resulted neuroprotective in a rotenone-induced rat model of PD counteracting a-synuclein translocation into the nuclei [41].

HDACi neuroprotection was further confirmed against neurotoxic pesticides and paraquat [37,42]. Indeed, trichostatin A selectively antagonizes mitochondrial fragmentation and cell death induced by MPP+ in human neuroblastoma cells [43], and sodium butyrate improves locomotor impairment and early mortality in a rotenoneinduced Drosophila model of PD [44], alleviates cognitive deficits in a rat model of PD in the pre-motor deficit stage [45], and up-regulates DJ-1 protein expression and protects neurons in cell cultures and mouse models against MPP+ toxicity $[46,47]$. DJ-1 is involved in the protection of oxidative stress, and mutation of $D J-1$ gene causes earlyonset PD [48]. These studies suggest the protective role for HDACi in PD models where the histone modifications is related to $\alpha$-synuclein, neurotoxic pesticides or paraquat.

\section{Amyotrophic lateral sclerosis}

ALS is a progressive neurodegenerative disease in which motor neurons in the brain and spinal cord are selectively destroyed. Usually, the disease manifests itself during the mid-50s, although there are rare cases of early-onset ALS. The symptoms of the disease are muscle wasting and atrophy leading to eventual paralysis and death [21]. ALS is typically fatal within 5 years of diagnosis due to a progressive, generalized paralysis that eventually affects the muscles of respiration, causing respiratory failure [21]. Areas where degenerating motor neurons are present in both ALS patients and mouse models are marked by the presence of cytokines and immune cells, including $\mathrm{T}$ cells, activated microglia, and astrocytes [21]. 
Although a generalized neuroinflammatory response may be driving progressive loss of motor neurons, not all inflammatory mediators have been strongly implicated in ALS. For instance, IL-1 $\beta$ may not be critical to ALS pathogenesis as genetic deletion of IL-1 $\beta$ does not change the lifespan or rate of motor neurodegeneration in mutant SOD-1 mice [21]. Currently, no effective pharmacological agents exist for the treatment of this devastating disease and neuroinflammation may accelerate the progression of ALS.

Epigenetic mechanisms are also involved in ALS because histone deacetlylases are shown to be aberrantly upregulated in neurons of brain from ALS patients and $\mathrm{HDACi}$ ameliorated disease progression [49-56].

Studies performed with sodium phenylbutyrate indicated that this histone deacetylase inhibitor significantly extended survival and improved both the clinical and neuropathological phenotypes in G93A transgenic ALS mice [50]. Also, the combined treatment of sodium phenylbutyrate and riluzole significantly extended survival and improved both the clinical and neuropathological phenotypes in G93A transgenic ALS mice more than either agent alone. In addition, riluzole/sodium phenylbutyrate treatment ameliorated gross lumbar and ventral horn atrophy, attenuated lumbar ventral horn neuronal cell death, and decreased reactive astrogliosis. Sodium phenylbutyrate/ riluzole administration increased acetylation at $\mathrm{H} 4$ and increased NFkappaB p50 translocation to the nucleus in G93A mice, consistent with a therapeutic effect [51]. A phase II study in ALS individuals revealed that sodium phenylbutyrate was safe and tolerable, and histone acetylation was significantly increased after sodium phenylbutyrate administration [52].

Similarly, the combination treatment of lithium and valproate (histone deacetylases inhibitor) produced a greater and more consistent effect in delaying the onset of disease symptoms, prolonging the life span and decreasing the neurological deficit scores, compared with the results of monotreatment with lithium or valproate [53], and treatment with trichostatin A [54], or valproate [55], delayed disease progression and/or increased survival in the SOD1-G93A mice. Conversely, a trial using valproic acid did not show a beneficial effect on survival or disease progression in patients with ALS [56].

\section{Huntington disease}

While neuroinflammation has been targeted in many neurodegenerative diseases ranging from $\mathrm{AD}$ to ALS to $\mathrm{PD}$, it has not received much attention from the $\mathrm{HD}$ community. However, several published trials, while not having neuroinflammation per se in mind, might have also targeted this process [57].

Several studies indicate that inflammation appears in the CNS during the progression of HD and HD-like pathology. Several brain regions from HD patients and controls revealed increased gliosis and expression of inflammation-related genes, including glial fibrillary acidic protein and complement proteins. Increases were most pronounced in the caudate putamen where brain pathology is most severe in HD patients [57]. Also, increased levels of pro-inflammatory cytokines involved in the innate immune response, such as IL-6, where detected in HD patients as well as an altered immune profile before onset of clinical HD symptoms, suggesting that striatal and cortical neurodegeneration could be exacerbated by inflammation [57]. Thus, it is clear that also HD is related to neuroinflammation process such as disruption of normal microglial functions and neuronal distress. This neuroinflammation may contribute to the death of additional neurons and once better understood, targeted interference with neuroinflammatory processes, active or reactive, could be a valuable tool for developing new therapeutic approaches [57].

Epigenetic research in Huntington's disease (HD), a neurodegenerative diseases caused by trinucleotide repeat expansion in the gene (HTT) coding for the huntingtin protein, revealed that mutant huntingtin directly interacts with HAT proteins, leading to altered histone acetylation $[58,59]$. Numerous studies revealed that treatment with HDACi arrested the ongoing progressive neuronal degeneration in both fly and mouse models of HD [60-64].

Chopra et al. have studied the in vivo efficacy of a brain-permeable sirtuin 2 inhibitor in two genetic mouse models of HD. AK-7 treatment improved motor function, extended survival, reduced brain atrophy and marked reduction of aggregated mutant huntingtin, a hallmark of HD pathology [61]. Since AK-7 treatment was beneficial in two HD mouse models, the data also suggests the therapeutic potential of sulfobenzoic acid derivatives in humans.

Another study report beneficial effects of the benzamide-type HDAC inhibitor, HDACi $4 \mathrm{~b}$, on disease phenotypes in N171-82Q transgenic mice, which include significantly improved movement and motor function, cognitive behavior and delayed weight loss over vehicletreated animals. Jia et al. [62] demonstrate that HDAC inhibition by $4 \mathrm{~b}$ can affect protein post-translational modification processes at the level of gene expression, can increase phosphorylation and acetylation of endogenous $\mathrm{Htt}$ protein and can prevent formation of mutant $\mathrm{Htt}$ aggregates in the brain; these effects may contribute to its clinically beneficial properties observed in HD mice. Because post-translational modification of target proteins can modify their accumulation, stability and/or clearance, we suggest that HDAC inhibitors may be acting to modify the processing of important proteins in the cell, such as the $\mathrm{Htt}$ protein [62]. Given the benefical effets of the above compounds on HD, other HDACi are under investigation $[63,64]$.

\section{Multiple sclerosis}

MS is a chronic condition in which the immune system attacks the axonal myelin sheaths. The site of inflammatory damage is scarred, thus the disease name is derived from sclerosis meaning "scar" in Latin [21]. Since these loci of injury can occur anywhere in the brain and spinal cord, the symptoms of the disease are usually diverse in different patients. These include fatigue, numbness, vision abnormalities, incontinence, muscle weakness, and paralysis.

MS is an autoimmune condition where foci of chronic inflammation lead to compromise of oligodendrocytes and destruction of the myelin sheath. This is followed by axonal damage and consequent neuronal degeneration. Inflammation and neurodegeneration do not occur simultaneously and axonal damage and brain atrophy may follow months after an acute innate immune response [21]. At today, numerous drugs target the immune system to reduce the progression of MS but they are only moderately effective, and the treatment of MS remains mostly symptomatic and far from satisfactory [21].

In the present review we have observed that aberrant regulation of acetylation homeostasis within neural cells might be a common pathogenetic mechanism underlying neurodegeneration. Histone acetyl transferases (HATs) and histone deacetylases (HDACs) finely tune cellular acetylation, targeting not only histones but also numerous proteins with key roles in cell metabolism, signaling and death. HDAC inhibitor are being evaluated currently in clinical trials for the above disorders and preclinical evidence suggests that pharmacological 
inhibition of HDACs is a promising therapeutic strategy also for the treatment of MS [65-67].

The effects of the HDACi trichostatin A and sodium phenylbutyrate were studied in the experimental allergic encephalomyelitis (EAE) an animal model of multiple sclerosis $[65,66]$. Trichostatin A or sodium phenylbutyrate treatment completely abrogates development of adoptive EAE $[65,66]$. Interestingly, clinical symptoms of EAE were much less in mice receiving sodium phenylbutyrate than control group. Histological and immunocytochemical analysis showed that sodium phenylbutyrate inhibited EAE-induced spinal cord mononuclear cell invasion and normalized iNOS, nitrotyrosine, and p65 (the RelA subunit of NF-kappaB) expression within the spinal cord. Taken together, our results raise the possibility that $r$ sodium phenylbutyrate may reduce the neuroinflammation and disease process in multiple sclerosis [66].

The effects of HDACi was also studied in another experimental model of chronic MS: C57BL/6 mice immunized with the myelin oligodendrocyte glycoprotein peptide $\mathrm{MOG}_{35-55}$ [67]. The study reports that the potent HDACi trichostatin A (TSA) while does not affect disease onset, reduces neurological impairment significantly. TSA treatments increases expression of neuroprotective proteins such as estrogen receptor- $\alpha$, insulin growth factor-2 (IGF-2), glutamate transporter EAAT2 and glutathione peroxidase, and decreases that of proapoptotic Bax, Bid, caspase-2 and apoptosis-inducing factor [67]. Furthermore, increased histone acetylation levels in the spinal cord of TSA-treated mice correlated with reduced levels of caspase 3 and 9 . The same study shows TSA also decreases expression of the chemokine macrophage inflammatory protein-2 (MIP-2) and splenocytes from TSA-treated mice show reduced proliferation to $\mathrm{MOG}_{35-55}$ as well as to nonspecific T-cell activators such as concavalin-A and phytohemoagglutinin [67]. These latter findings clearly indicate that TSA treatment severely affects development of the auto-immune response in MS.

\section{Recent studies on neuroprotective mechanism of HDAC inhibitors on neurodegenerative disorders}

In this review, we have shown that HDAC inhibitors have neuroprotective, neurotrophic and anti-inflammatory properties in neurodegenerative disorders. Now, we discuss the targets and mechanisms underlying these HDACi effects indicating their potential clinical effecacy in treating neurodegenerative disorders.

\section{HDACi Neuroprotection against oxidative stress induced by glutathione depletion}

Oxidative stress is believed to be an important mediator of neurodegeneration. However, the transcriptional pathways induced in neurons by oxidative stress that activate protective gene responses have yet to be fully delineated. Ryu et al. [68] report that the transcription factor Sp1 is acetylated in response to oxidative stress in neurons. Histone deacetylase (HDAC) inhibitors augment Sp1 acetylation, Sp1 DNA binding, and Sp1-dependent gene expression and confer resistance to oxidative stress-induced death in vitro and in vivo. Sp1 activation is necessary for the protective effects of HDAC inhibitors [68]. Together, these results demonstrate that HDAC inhibitors inhibit oxidative death independent of polyglutamine expansions by activating an Sp1-dependent adaptive response. These findings suggest that these agents are able to abrogate the deleterious effects of oxidative stress in neurons independent of expanded polyglutamine repeats and thus may be propitious therapeutic agents for a host of neurological diseases, including Huntington's disease, amyotrophic lateral sclerosis,
Parkinson's disease, and stroke, which have been associated in some cases with decreased histone acetyl transferase activity and in all cases with increased levels of oxidative damage [68].

\section{HDACi neuroprotection against neuronal oxidative stress- induced death}

Langley et al. [69] demonstrated that pulse exposure of cortical neurons $(2 \mathrm{~h})$ in an in vitro model of oxidative stress results in durable neuroprotection by HDACi. Protection was associated with transcriptional upregulation of the cell cycle inhibitor, p21 $21^{\text {wafl/cip1 }}$, both in this model and in an in vivo model of permanent ischemia. Transgenic overexpression of $\mathrm{p} 21^{\text {wafl/cip } 1}$ in neurons can mimic the protective effect of HDAC inhibitors against oxidative stress-induced toxicity, including death induced by glutathione depletion or peroxide addition [69]. The protective effect of $\mathrm{p} 21^{\text {wafl/cipl }}$ in the context of oxidative stress appears to be unrelated to its ability to act in the nucleus to inhibit cell cycle progression. However, although $\mathrm{p} 21^{\text {wafl }} /$ ${ }^{\text {cip1 }}$ is sufficient for neuroprotection, it is not necessary for HDAC inhibitor neuroprotection, because these agents can completely protect neurons cultured from $\mathrm{p} 21^{\text {wafl/cip1 }}$-null mice. Together these findings demonstrate (1) that pulse inhibition of HDACs in cortical neurons can induce neuroprotection without apparent toxicity; (2) that $\mathrm{p} 21^{\text {wafl } 1 /}$ ${ }^{\text {cip1 }}$ is sufficient but not necessary to mimic the protective effects of HDAC inhibition; and (3) that oxidative stress in this model induces neuronal cell death via cell cycle-independent pathways that can be inhibited by a cytosolic, noncanonical action of $\mathrm{p} 21^{\text {wafl/cip1 }}$.

In this study, the authors show that HDAC inhibitor toxicity can be abrogated without lessening the neuroprotective effect, implying that the neuroprotective and toxic effects of HDAC inhibition are separable [69]. Understanding the neuroprotective versus toxic mechanisms induced by HDAC inhibition, including which HDACs are involved in the different processes, will be important as this class of drugs are considered for and move toward the clinical treatment of neurodegenerative disease.

\section{HDCi neuroprotection against glutamate-induced cytotoxicity}

Emerging evidence suggests that alpha-synuclein (alpha-syn), which is traditionally thought to have a pathophysiological role in neurodegenerative diseases, can have neuroprotective effects. A recent study investigated whether endogenous alpha-syn in neurons can be induced by valproic acid (VPA), a mood-stabilizer, anticonvulsant and histone deacetylase (HDAC) inhibitor, and if so, whether the alpha-syn induction is neuroprotective [70]. VPA treatment of rat cerebellar granule cells caused a robust dose- and time-dependent increase in levels of alpha-syn protein and mRNA and in the intensity of alpha-syn immunostaining. Knockdown of VPA-induced alphasyn overexpression with alpha-syn antisense oligonucleotides or siRNA completely blocked VPA-induced neuroprotection. alpha-Syn knockdown also exacerbated glutamate neurotoxicity, stimulated the expression of the proapoptotic gene ubiquitin-conjugating enzyme $\mathrm{E} 2 \mathrm{~N}$, and downregulated the expression of the anti-apoptotic gene Bcl2 [70]. Induction of alpha-syn by VPA was associated with inhibition of HDAC activity, resulting in hyperacetylation of histone $\mathrm{H} 3$ in the alphasyn promoter and a marked increase in alpha-syn promoter activity. Moreover, VPA-induced alpha-syn induction and neuroprotection were mimicked by HDAC inhibitors sodium 4-phenylbutyrate and trichostatin A (TSA) [70]. alpha-syn was also induced by VPA in rat cerebral cortical neurons. Additionally, treatment of rats with VPA, sodium butyrate, or TSA markedly increased alpha-syn protein levels 
in the cortex and cerebellum. Together, our results demonstrate for the first time that VPA induces alpha-syn in neurons through inhibition of HDAC and that this alpha-syn induction is critically involved in neuroprotection against glutamate excitotoxicity [70]. Clinically, VPA may represent a suitable treatment for excitotoxicity-related neurodegenerative diseases.

\section{HDACi neuroprotection against tauopathies}

Tauopathies are neurodegenerative disorders for which there are no effective treatments. Some disorders are caused by mutations in tau that increase the probability of tau aggregate formation, leading to intracellular neurofibrillary tangles. These disorders are typically referred to as fronto-temporal dementias. Other tauopathies occur in different brain regions (corticobasal syndrome, progressive supranuclear palsy, and so forth) [71]. Tau pathology is associated with a number of age-related neurodegenerative disorders and few treatments have been demonstrated to diminish the impact of tau pathology in mouse models and none are yet effective in humans. Histone deacetylase 6 (HDAC6) is an enzyme that removes acetyl groups from cytoplasmic proteins, rather than nuclear histones. Its substrates include tubulin, heat shock protein 90 and cortactin. Tubastatin A is a selective inhibitor of HDAC6. Modification of tau pathology by specific inhibition of HDAC6 presents a potential therapeutic approach in tauopathy [71]. Potential mechanisms by which HDAC6 inhibitors might benefit the rTg4510 mouse include stabilization of microtubules secondary to increased tubulin acetylation, increased degradation of tau secondary to increased acetylation of HSP90 or both. These data support the use of HDAC6 inhibitors as potential therapeutic agents against tau pathology [71].

Also, the accumulation of hyperphosphorylated tau in neurofibrillary tangles (NFTs) is a neuropathological hallmark of tauopathies and another study described a novel mechanism in which the acetylation of tau on KXGS motifs inhibits phosphorylation on this same motif, and also prevents tau aggregation [72]. Using a site-specific antibody to detect acetylation of KXGS motifs, the authors demonstrated that these sites are hypoacetylated in patients with $\mathrm{AD}$, as well as a mouse model of tauopathy, suggesting that loss of acetylation on KXGS motifs renders tau vulnerable to pathogenic insults. Furthermore, they identify histone deacetylase 6 (HDAC6) as the enzyme responsible for the deacetylation of these residues, and provide proof of concept that acute treatment with a selective and blood-brain barrier-permeable HDAC6 inhibitor enhances acetylation and decreases phosphorylation on tau's KXGS motifs in vivo [72]. As such, we have uncovered a novel therapeutic pathway that can be manipulated to block the formation of pathogenic tau species in disease.

A further study tested the potential of two selective HDAC6 inhibitors, tubastatin A and ACY-1215, to reduce tau hyperphosphorylation in a mouse model of $\mathrm{AD}$ [73]. The authors found that both tubastatin A and ACY-1215 alleviated behavioral deficits, altered amyloid- $\beta$ (A $\beta)$ load, and reduced tau hyperphosphorylation in AD mice without obvious adverse effects [73]. These data suggested that tubastatin A and ACY-1215 not only promoted tubulin acetylation, but also reduced production and facilitated autophagic clearance of $A \beta$ and hyperphosphorylated tau. Further, the decreased hyperphosphorylated tau and increased tubulin acetylation may account for the improved microtubule stability in AD mice after tubastatin A/ACY-1215 treatment. These preclinical results support the detrimental role of HDAC6 in AD, and offer prospective approaches for using tubastatin $\mathrm{A} / \mathrm{ACY}-1215$ as potential therapeutic strategy for $\mathrm{AD}$ [73].

\section{HDACi neuroprotection by the induction of the expression of neurotrophins}

The evidence, reported in the present review, supports the notion that histone hypoacetylation and transcriptional dysfunction are involved in a large number of neurodegenerative disorders and treatment with HDAC inhibitors protects against neurodegeneration. Multiple genes regulated by HDAC inhibition and involved in neuroprotection and neurotrophicity have been identified [74].

The HDAC restoring effects appear to be mediated by multiple HDAC-regulated gene products including BDNF, GDNF, HSP70, a-synuclein, Bcl-2, Bcl- $\mathrm{X}_{\mathrm{L}}, \mathrm{p} 21$, and gelsolin, among others [74]. Nontranscriptional effects of HDAC inhibitors, such as hyperacetylation and stabilization of microtubule proteins, have also been shown in many neurodegenerative disease models. Studies suggest that HDAC inhibitors have neuroprotective, neurotrophic, and anti-inflammatory effects, as well as improve neurological performance and learning/ memory in various neurodegenerative conditions. HDAC inhibitioninduced neurotrophins were found not only in neurons, but also in astrocytes, suggesting that glia are also an important target for therapeutic intervention [74].

\section{Conclusions}

As noted above, HDAC inhibitors suppress neuroinflammation by inhibiting microglia activation in cultured cells and stroke models. This raises the possibility that HDAC inhibitors may be developed as anti-inflammatory drugs in the treatment of brain disorders. This is an important area for future research in view that presently there is no clinical drug that robustly mitigates neuroinflammation in the brain. In addition to transcriptional regulation, non-transcriptional events, such as improvement of microtubule stability via enhanced acetylation, have been implicated in the neuroprotective effects elicited by HDAC inhibition in a number of disease models. HAT-mediated acetylation of HTT is an important mechanism for removing accumulated toxic protein in the HD model. It remains to be investigated whether this is a general mechanism for selective trafficking of proteins for lysosomal degradation and clearance, and whether this is a regulatory target of HDAC inhibitors.

\section{References}

1. Tsankova N, Renthal W, Kumar A, Nestler EJ (2007) Epigenetic regulation in psychiatric disorders. Nat Rev Neurosci 8: 355-367. [Crossref]

2. Mehler MF (2008) Epigenetic principles and mechanisms underlying nervous system functions in health and disease. Prog Neurobiol 86: 305-341. [Crossref]

3. Rodenhiser D, Mann M (2006) Epigenetics and human disease: translating basic biology into clinical applications. CMAJ 174: 341-348.

4. Kouzarides T (2007) Chromatin modifications and their function. Cell 128: 693-705. [Crossref]

5. Jenuwein T, Allis CD (2001) Translating the histone code. Science 293: 1074-1080. [Crossref]

6. Wood MA, Hawk JD, Abel T (2006) Combinatorial chromatin modifications and memory storage: a code for memory? Learn Mem 13: 241-244. [Crossref]

7. Levenson JM, Sweatt JD (2005) Epigenetic mechanisms in memory formation. Nat Rev Neurosci 6: 108-118.

8. Carey N, La Thangue NB (2006) Histone deacetylase inhibitors: gathering pace. Curr Opin Pharmacol 6: 369-375. [Crossref]

9. Minucci S, Pelicci PG (2006) Histone deacetylase inhibitors and the promise of epigenetic (and more) treatments for cancer. Nat Rev Cancer 6: 38-51. [Crossref]

10. Saha RN, Pahan K (2006) HATs and HDACs in neurodegeneration: a tale of disconcerted acetylation homeostasis. Cell Death Differ 13: 539-550. [Crossref] 
11. Wang Y, Wang X, Liu L, Wang X (2009) HDAC inhibitor trichostatin A-inhibited survival of dopaminergic neuronal cells. Neurosci Lett 467: 212-216. [Crossref]

12. Rouaux C, Jokic N, Mbebi C, Boutillier S, Loeffler J. P, et al. (2003) Critical loss of $\mathrm{CBP} / \mathrm{p} 300$ histone cetylase activity by caspase-6 during neurodegeneration. EMBO J 22: 6537-6549.

13. Pirooznia SK, Sarthi J, Johnson AA, Toth MS, Chiu K, et al. (2012) Tip60 HAT activity mediates APP induced lethality and apoptotic cell death in the CNS of a Drosophila Alzheimer's disease model. PLOS ONE 7: e41776.

14. Steffan JS, Bodai L, Pallos J, Poelman M, McCampbell A, et al. (2001) Histone deacetylase inhibitors arrest polyglutamine-dependent neurodegeneration in Drosophila. Nature 413: 739-743.

15. Jakovcevski M, Akbarian S (2012) Epigenetic mechanisms in neurological disease. Nat Med 18: 1194-1204. [Crossref]

16. Desplats P, Spencer B, Coffee E, Patel P, Michael S, et al. (2011) Alpha-synuclein sequesters Dnmt1 from the nucleus: a novel mechanism for epigenetic alterations in Lewy body diseases. J Biol Chem 286: 9031-9037.

17. Ryu H, Lee J, Hagerty SW, Soh BY, McAlpin SE, et al. (2006) ESET/SETDB1 gene expression and histone H3 (K9) trimethylation in Huntington's disease. Proc Natl Acad Sci U S A 103: 19176-19181. [Crossref]

18. Rajendran P, Ho E, Williams DE, Dashwood RH (2011) Dietary phytochemicals, HDAC inhibition, and DNA damage/repair defects in cancer cells. Clin Epigenetics 3: 4. [Crossref]

19. Dal Piaz F, Tosco A, Eletto D, Piccinelli AL, Moltedo O, et al. (2010) The Identification of a Novel Natural Activator of p300 Histone Acetyltranferase Provides New Insights into the Modulation Mechanism of this Enzyme. ChemBioChem 11: 818-827.

20. Coppedè $F(2014)$ The potential of epigenetic therapies in neurodegenerative diseases. Front Genet 5: 220. [Crossref]

21. Pizza V, Agresta A, D'Acunto CW, Festa M, Capasso A (2011) Neuroinflamm-aging and neurodegenerative diseases: an overview. CNS Neurol Disord Drug Targets 10: 621-634. [Crossref]

22. Migliore L, Coppedè F (2009) Genetics, environmental factors and the emerging role of epigenetics in neurodegenerative diseases. Mutat Res 667: 82-97. [Crossref]

23. Zhang K, Schrag M, Crofton A, Trivedi R, Vinters H, et al. (2012) Targeted proteomics for quantification of histone acetylation in Alzheimer's disease. Proteomics 12: 12611268. [Crossref]

24. Ding H, Dolan PJ, Johnson GV (2008) Histone deacetylase 6 interacts with the microtubule-associated protein tau. J Neurochem 106: 2119-2130.

25. Gräff J, Rei D, Guan JS, Wang WY, Seo J, et al. (2012) An epigenetic blockade of cognitive functions in the neurodegenerating brain. Nature 483: 222-226. [Crossref]

26. Coppedè $F$ (2014) Advances in the genetics and epigenetics of neurodegenerative diseases. Epigenetics Neurodegener Dis 1: 3-31.

27. Coppieters N, Dieriks BV, Lill C, Faull RL, Curtis MA, et al. (2014) Global changes in DNA methylation and hydroxymethylation in Alzheimer's disease human brain. Neurobiol Aging 35: 1334-1344.

28. Karagiannis TC, Ververis K (2012) Potential of chromatin modifying compounds for the treatment of Alzheimer's disease. Pathobiol Aging Age Relat Dis 2: 14980.

29. Fischer A, Sananbenesi F, Wang X, Dobbin M, Tsai LH (2007) Recovery of learning and memory is associated with chromatin remodelling. Nature 447: 178-182. [Crossref]

30. Ricobaraza A, Cuadrado-Tejedor M, Marco S, Pérez-Otaño I, García-Osta A (2010) Phenylbutyrate rescues dendritic spine loss associated with memory deficits in a mouse model of Alzheimer disease. Hippocampus 22: 1040-1050.

31. Govindarajan N, Agis-Balboa RC, Walter J, Sananbenesi F, Fischer A (2011) Sodium butyrate improves memory function in an Alzheimer's disease mouse model when administered at an advanced stage of disease progression. J Alzheimers Dis 26: 187-197.

32. Francis YI, Fà M, Ashraf H, Zhang H, Staniszewski A, et al. (2009) Dysregulation of histone acetylation in the APP/PS1 mouse model of Alzheimer's disease. J Alzheimers Dis 18: 131-139. [Crossref]

33. Kilgore M, Miller CA, Fass DM, Hennig KM, Haggarty SJ, et al. (2010) Inhibitors of class 1 histone deacetylases reverse contextual memory deficits in a mouse model of Alzheimer's disease. Neuropsychopharmacology 35: 870-880.

34. Zhang Z, Schluesener YHJ (2013) Oral administration of histone deacetylase inhibitor MS-275 ameliorates neuroinflammation and cerebral amyloidosis and improves behavior in a mouse model. J Neuropathol Exp Neurol 72: 178-185.
35. Sung YM, Lee T, Yoon H, Di Battista AM, Song J, Sohn Y, et al. (2013) Mercaptoacetamide-based class II HDAC inhibitor lowers Aß levels and improves learning and memory in a mouse model of Alzheimer's disease. Exp. Neurol 239, 192-201.

36. Banati RB, Daniel SE, Blunt SB (1998) Glial pathology but absence of apoptotic nigral neurons in long-standing Parkinson's disease. Mov. Disord 13, 221-227.

37. Coppedè F (2012) Genetics and epigenetics of Parkinson's disease ScientificWorldJournal 2012: 489830. [Crossref]

38. Harrison IF, Dexter DT (2013) Epigenetic targeting of histone deacetylase: therapeutic potential in Parkinson's disease? Pharmacol Ther 140: 34-52.

39. Goers J, Manning-Bog AB, Mc Cormack AL, Millett IS, Doniach S, et al. (2003) Nuclear localization of alpha-synuclein and its interaction with histones. Biochemistry 42: 8465-8471.

40. Kontopoulos E, Parvin JD, Feany MB (2006) Alpha-synuclein acts in the nucleus to inhibit histone acetylation and promote neurotoxicity. Hum Mol Genet 15: 3012-3023. [Crossref]

41. Outeiro TF, Kontopoulos E, Altmann SM, Kufareva I, Strathearn KE, et al. (2007) Sirtuin 2 inhibitors rescue alpha-synuclein-mediated toxicity in models of Parkinson's disease. Science 317: 516-519.

42. Monti B, Gatta V, Piretti F, Raffaelli SS, Virgili M, et al. (2010) Valproic acid is neuroprotective in the rotenone rat model of Parkinson's disease: involvement of alphasynuclein. Neurotox Res 17: 130-141. [Crossref]

43. Song C, Kanthasamy A, Anantharam V, Sun F, Kanthasamy AG (2010) Environmental neurotoxic pesticide increases histone acetylation to promote apoptosis in dopaminergic neuronal cells: relevance to epigenetic mechanisms of neurodegeneration. Mol Pharmacol 77: 621-632.

44. Zhu M, Li W.W, Lu CZ (2014) Histone decacetylase inhibitors prevent mitochondrial fragmentation and elicit early neuroprotection against MPP+. CNS Neurosci Ther 20: 308-316.

45. St Laurent R, O'Brien LM, Ahmad ST (2013) Sodium butyrate improves locomotor impairment and early mortality in a rotenone-induced Drosophila model of Parkinson's disease. Neuroscience 246: 382-390.

46. Rane P, Shields J, Heffernan M, Guo Y, Akbarian S, King JA (2012) The histone deacetylase inhibitor, sodium butyrate, alleviates cognitive deficits in pre-motor stage PD. Neuropharmacology 62, 2409-2412.

47. Zhou W, Bercury K, Cummiskey J, Luong N, Lebin J, et al. (2011) Phenylbutyrate upregulates the DJ-1 protein and protects neurons in cell culture and in animal models of Parkinson disease. J Biol Chem 286: 14941-14951.

48. Bonifati V, Rizzo P, van Baren MJ, Schaap O, Breedveld GJ, et al. (2003) Mutations in the DJ-1 gene associated with autosomal recessive early-onset parkinsonism. Science 299: 256-259.

49. Janssen C, Schmalbach S, Boeselt S, Sarlette A, Dengler R, et al. (2010) Differential histone deacetylase mRNA expression patterns in amyotrophic lateral sclerosis. $J$ Neuropathol Exp Neurol 69: 573-581.

50. Ryu H, Smith K, Camelo SI, Carreras I, Lee J, Iglesias AH, et al. (2005) Sodium phenylbutyrate prolongs survival and regulates expression of anti-apoptotic genes in transgenic amyotrophic lateral sclerosis mice. J Neurochem 93: 1087-1098.

51. Del Signore SJ, Amante DJ, Kim J, Stack EC, Goodrich S, et al. (2009) Combined riluzole and sodium phenylbutyrate therapy in transgenic amyotrophic lateral sclerosis mice. Amyotroph Lateral Scler 10: 85-94.

52. Cudkowicz ME, Andres PL, Macdonald SA, Bedlack RS, Choudry R, et al. (2009) Phase 2 study of sodium phenylbutyrate in ALS. Amyotroph. Lateral Scler 10: 99-106.

53. Feng HL, Leng Y, Ma CH, Zhang J, Ren M, Chuang DM (2008) Combined lithium and valproate treatment delays disease onset, reduces neurological deficits and prolongs survival in an amyotrophic lateral sclerosis mouse model. Neuroscience 155: 567-572.

54. Yoo YE, Ko CP (2011) Treatment with trichostatin A initiated after disease onset delays disease progression and increases survival in a mouse model of amyotrophic lateral sclerosis. Exp Neurol 231: 147-159.

55. Sugai F, Yamamoto Y, Miyaguchi K, Zhou Z, Sumi H, et al. (2004) Benefit of valproic acid in suppressing disease progression of ALS model mice. Eur J Neurosci 20: 31793183. [Crossref]

56. Piepers S, Veldink JH, de Jong SW, van der Tweel I, van der Pol WL, et al. (2009) Randomized sequential trial of valproic acid in amyotrophic lateral sclerosis. Ann Neurol 66: 227-234. [Crossref] 
57. Möller T (2010) Neuroinflammation in Huntington's disease. J Neural Transm (Vienna) 117: 1001-1008. [Crossref]

58. Steffan JS, Kazantsev A, Spasic-Boskovic O, Greenwald M, Zhu YZ, et al. (2000) The Huntington's disease protein interacts with p53 and CREB-binding protein and represses transcription. Proc Natl Acad Sci USA 97: 6763-6768.

59. Jiang H, Poirier M.A, Liang Y, Pei Z, Weiskittel CE, et al. (2006) Depletion of CBP is directly linked with cellular toxicity caused by mutant huntingtin. Neurobiol Dis 23 : 543-551.

60. Gray SG (2010) Targeting histone deacetylases for the treatment of Huntington's disease. CNS Neurosci Ther 16: 348-361. [Crossref]

61. Chopra V, Quinti L, Kim J, Vollor L, Narayanan KL, et al. (2012) The sirtuin 2 inhibitor AK-7 is neuroprotective in Huntington's disease mouse models. Cell Rep 2: 1492-1497.

62. Jia H, Kast RJ, Steffan JS, Thomas EA (2012) Selective histone deacetylase (HDAC) inhibition imparts beneficial effects in Huntington's disease mice: implications for the ubiquitin-proteasomal and autophagy systems. Hum Mol Genet 21: 5280-5293.

63. Bürli RW, Luckhurst CA, Aziz O, Matthews KL, Yates D, et al. (2013) Design, synthesis, and biological evaluation of potent and selective class IIa histone deacetylase (HDAC) inhibitors as a potential therapy for Huntington's disease. J Med Chem 56: 9934-9954.

64. Duan W (2013) Targeting sirtuin-1 in Huntington's disease: rationale and current status. CNS Drugs 27: 345-352. [Crossref]

65. Gray SG, Dangond F (2006) Rationale for the use of histone deacetylase inhibitors as a dual therapeutic modality in multiple sclerosis. Epigenetics 1: 67-75.
66. Dasgupta S, Zhou Y, Jana M, Banik NL, Pahan K (2003) Sodium phenylacetate inhibits adoptive transfer of experimental allergic encephalomyelitis in $\mathrm{SJL} / \mathrm{J}$ mice at multiple steps. J Immunol 170: 3874-3882.

67. Camelo S, Iglesias, A.H, Hwang D, Due B, Ryu H, Smith, K. et al. (2005) Transcriptional therapy with the histone deacetylase inhibitor trichostatin A ameliorates experimental autoimmune encephalomyelitis. J Neuroimmunol 164: 10-21.

68. Ryu H, Lee J, Olofsson BA, Mwidau A, Dedeoglu A, et al. (2003) Histone deacetylase inhibitors prevent oxidative neuronal death independent of expanded polyglutamine repeats via an Sp1-dependent pathway. Proc. Natl Acad Sci USA 100: 4281-4286.

69. Langley B, D'Annibale MA, Suh K, Ayoub I, Tolhurst A, et al. (2008) Pulse inhibition of histone deacetylases induces complete resistance to oxidative death in cortical neurons without toxicity and reveals a role for cytoplasmic p21(wafl/cip1) in cell cycle-independent neuroprotection. J Neurosci 28: 163-176.

70. Leng Y, Chuang DM (2006) Endogenous a-synuclein is induced by valproic acid through histone deacetylase inhibition and participates in neuroprotection against glutamate-induced excitotoxicity. $J$ Neurosci 26: 7502-7512.

71. Selenica ML, Benner L, Housley SB, Manchec B, Lee DC, et al. (2014) Histone deacetylase 6 inhibition improves memory and reduces total tau levels in a mouse model of tau deposition. Alzheimers Res 6: 12.

72. Cook C, Carlomagno Y, Gendron TF, Dunmore J, Scheffel K, et al. (2014) Acetylation of the KXGS motifs in tau is a critical determinant in modulation of tau aggregation and clearance. Hum Mol Genet 23: 104-116. [Crossref]

73. Zhang L, Liu C, Wu J, Tao JJ, Sui XL, Yao ZG, et al. (2014) Tubastatin A/ACY-1215 improves cognition in alzheimer's disease transgenic mice. J Alzheimers Dis 41: 1193-1205.

74. Chuang DM, Leng Y, Marinova Z, Kim HJ, Chiu CT (2009) Multiple roles of HDAC inhibition in neurodegenerative conditions. Trends Neurosci 32: 591-601. [Crossref]

Copyright: $\odot 2017$ Milano W. This is an open-access article distributed under the terms of the Creative Commons Attribution License, which permits unrestricted use, distribution, and reproduction in any medium, provided the original author and source are credited. 